\title{
El programa de asistencia policial de la AID en Uruguay (1965-1974) ${ }^{1}$
}

CLARA ALDRIGHI*

Resumo: Este artigo analisa a ingerência dos Estados Unidos na política interna do Uruguai nos anos que precederam o golpe de Estado de 1973; documenta o papel decisivo exercido pelo Programa de Segurança Pública da AID na repressão aos movimentos sociais e guerrilheiros, e o apoio à política autoritária que culminou com a implantação da ditadura.

Abstract: This article analyzes the interference of the United States in Uruguayan internal politics throughout the years that preceded the military coup; it documents the decisive role played by the USAID's Public Safety Program in the repression of the social and guerrilla movements, and sponsoring the authoritarian policies that culminated in the dictatorial regime.

Palavras chave: Uruguai. Contra-insurgência. Assistência policial.

Key words: Uruguay. Counterinsurgency. Police assistance.

\section{La "primera línea de defensa"}

El programa de ayuda a las policías civiles de todo el mundo fue creado en 1955 por voluntad del presidente Dwight Eisenhower, mediante una resolución del Consejo de Seguridad Nacional. Durante los primeros años fue conocido como Programa 1290-D; posteriormente adoptó el nombre de Programa de Seguridad Pública (PSP). Su objetivo era proveer asistencia y entrenamiento a las Policías de los países en vías de desarrollo, para que lograran combatir eficazmente "la subversión y el terror comunistas". ${ }^{2}$

* Facultad de Humanidades y Ciencias de la Educación, Instituto de Historia, Universidad de la República.

1 Una versión ampliada de este artículo ha sido publicada en mi libro La intervención de Estados Unidos en Uruguay (1965-1973). Tomo 1. El caso Mitrione. Montevideo: Trilce, 2007.

2 Departamento de Estado, OPS/AID Washington, "Terrorism, a National and International Problem. The Office of Public Safety Seminar", 15.9.1973; "Discussion of OPS Role" por Laurent J. Goin, en National Archives and Records Administration, Washington DC (NARA), Record Group (RG) 286, box 10.

Estudos Ibero-Americanos, PUCRS, v. XXXIV, n. 1, p. 181-204, junho 2008 
Fue ubicado en la jurisdicción del Departamento de Estado y su gestión encomendada a la Administración para la Cooperación Internacional (ICA), predecesora de la Agencia para el Desarrollo Internacional (AID). La primera misión de Seguridad Pública fue establecida en 1955 en la Indonesia de Sukarno. Poco después otra fue instalada en Vietnam del Sur, bajo directo control de la CIA. En agosto de 1962 el presidente John F. Kennedy proporcionó un decisivo impulso al Programa - que prestaba asistencia a las Policías de 26 países - creando en la AID un organismo semiautónomo, la Oficina de Seguridad Pública (OPS), y asignándole mayores recursos. El secretario de Justicia, Robert Kennedy, fue el principal promotor de la conversión de las Policías aliadas en un elemento clave de la política contrainsurgente de Estados Unidos. ${ }^{3}$

Para dirigir la OPS fue designado un hombre de la CIA, Byron Engle, ex administrador de programas clandestinos y funcionario de la Agencia desde su fundación en 1947. Aunque su nombramiento fue resistido por el Departamento de Estado, Engle traía consigo una sólida experiencia y considerable prestigio. Entre sus méritos se hallaban la dirección del adiestramiento de la Policía japonesa al término de la segunda guerra mundial y el asesoramiento a las fuerzas policiales de Turquía. El FBI consideró negativamente su designación y no se mostró dispuesto a cooperar. Su director J. Edgard Hoover llegó a comentar que el PSP "era una cobertura más de la CIA y no pensaba malgastar su sangre vital en una competencia burocrática". ${ }^{4}$

El Programa de Seguridad Pública era considerado un complemento o auxiliar del de Asistencia Militar (MAP). Los cometidos de ambos programas sustancialmente coincidían: preparar fuerzas

3 MAECHLING Charles. Contrainsurgencia: la primera prueba de fuego. En: KLARE, Michael; y KORNBLUH, Peter (coord.). Contrainsurgencia, proinsurgencia y antiterrorismo en los 80. El arte de la guerra de baja intensidad, México: Grijalbo, 1990, p. 33-64; KLARE, Michael; y STEIN, Nancy. La exportación de instrumentos de represión. En: KLARE, Michael; y STEIN Nancy. Armas y poder en América Latina. México: ERA, 1978, p. 171; Memorando de Byron Engle, director OPS/AID, Washington, "Request by New York Times for Access to Information Concerning US Public Safety Program in Uruguay", 18.8.1970, NARA.RG286, box 109. El número de países asistidos por el PSP a mediados de 1961 es tomado de McCLINTOCK Michael. Instruments of Statecraft: US Guerilla Warfare, Counterinsurgency and Counterterrorism 1940-1990. Nueva York: Pantheon Books, 1992, cap."The Apperatus in the Field", reproducido en www.statecraft.org, p.5 y nota 51 (donde se cita un documento del Departamento de Estado de junio de 1962). Klare y Stein indican al respecto otra cifra: 39 países asistidos por el Programa en 1962 (op. cit., p. 171).

4 LANGGUTH, A. J. Hidden Terrors. The Truth About US Police Operation in Latin America. New York: Pantheon Books, 1978, p. 47-49; MAECHLING, op. cit., p. 46. 
locales en condiciones de llevar a cabo operaciones de contrainsurgencia. Armadas y equipadas por Estados Unidos, dirigidas por oficiales entrenados por Estados Unidos y asesoradas por expertos norteamericanos. En 1971, durante la primera presidencia de Richard Nixon, el subsecretario de Estado U. Alexis Johnson explicaba las diferencias existentes entre el MAP y el PSP: "La labor policial efectiva es como la 'medicina preventiva'. La Policía puede ocuparse de las amenazas al orden interno en sus etapas de formación. Si no estuviera preparada para hacerlo, se requeriría de una 'cirugía mayor' para remediar estas amenazas, y una acción así es dolorosa, cara y desgarradora". ${ }^{5}$

En 1974, en vísperas de su clausura, la OPS prestaba ayuda a las Policías de 40 Estados del Tercer Mundo y disponía de un presupuesto anual que superaba los 50 millones de dólares. Cuatrocientos consejeros de Seguridad Pública - en rigor, instructores policiales - residían en el extranjero para dirigir directamente la capacitación. ${ }^{6}$ El PSP era utilizado por la CIA para encubrir a sus funcionarios e impulsar sus propios objetivos. Un documento secreto del Departamento de Estado, elaborado en 1962, admitía la participación de la Agencia en el PSP: "La CIA tiene personal integrado en los programas policiales de la AID. [...]. La CIA tiene la responsabilidad, implícita en su estatuto, de fortalecer la capacidad antisubversiva de las fuerzas de Policía extranjeras. [...]. La CIA provee la última información de los desarrollos de la estrategia y las técnicas chino-soviéticas a su personal en los programas policiales de la AID, que trabajan como consejeros en contrainsurgencia, contraespionaje, contraguerrilla y otros campos antisubversivos. [...]. La CIA financia y dirige los programas de asistencia policial en Turquía, Thailandia e Indonesia en sus aspectos oficiales y clandestinos, en el desarrollo de mecanismos de investigación capaces de detectar individuos $u$ organizaciones subversivas, en la recopilación y procesamiento de información [...] y en la neutralización de sus esfuerzos". ${ }^{7}$

5 KLARE y STEIN, La política económica de las ventas norteamericanas de armamento a Latinoamérica, en: ibid., Armas y poder..., op. cit., p. 23.

6 KLARE y STEIN, La exportación de instrumentos de represión, en: ibid., Armas y poder..., p. 171-172.

7 Departamento de Estado, "Interdepartmental Technical Subcommittee on Police Advisory Assistance Programs", 11.6.1962, p. 16, cit. por McCLINTOCK, op. cit., p. 4, notas 48, 54; McSHERRY, Patrice. Predatory States. Operation Condor and Cover War in Latin America. Oxford: Rowman \& Littlefield, 2005, p. 15. 
En noviembre de 1970 David Fairchild, ex oficial de la AID que había servido en República Dominicana, declaró que la CIA había utilizado la misión de Seguridad Pública, integrada por 18 consejeros, para encubrir a seis de sus funcionarios. ${ }^{8}$ Philip Agee reveló la identidad de dos hombres de la CIA con adscripción ficticia en el PSP: Robert Weatherwax, que actuó en Ecuador en 1960 como consejero de Seguridad Pública de la ICA, y William Cantrell, consejero de investigaciones en Uruguay entre setiembre de 1966 y marzo de $1970 .{ }^{9}$ El policía salvadoreño José Alberto Medrano reveló que si bien la OPS había sido la responsable de la creación de la Agencia Nacional de Seguridad de su país, "las labores cotidianas y más importantes de inteligencia son coordinadas por la CIA". ${ }^{10}$

Al ser cancelado el Programa en 1975, se conocieron los balances y estadísticas de la ayuda proporcionada por la OPS. Se habían distribuido 200 millones de dólares en armas y equipos; 10.000 policías extranjeros habían recibido adiestramiento en la Academia Internacional de Policía (IPA) y otras escuelas de Estados Unidos; un millón de policías, bajo la dirección de los consejeros de Seguridad Pública, habían sido adiestrados en los países participantes del Programa. ${ }^{11}$

La asistencia más sustanciosa fue destinada a regímenes proestadounidenses asediados por ataques revolucionarios: Vietnam del Sur, Thailandia, Filipinas, Guatemala, Brasil y Uruguay. ${ }^{12}$ En los doce años de existencia de la OPS, siete consejeros de Seguridad Pública fueron ejecutados por las fuerzas insurgentes: seis en Vietnam y uno en Uruguay. ${ }^{13}$

Cuando murió Daniel Anthony Mitrione, consejero jefe de la división de Seguridad Pública de Uruguay, ejecutado por el Movimiento de Liberación Nacional Tupamaros (MLN) el 9 de agosto de 1970, las misiones del PSP estaban instaladas en 15 países de América Latina y los consejeros residentes eran, según

8 KLARE, Michael; y STEIN Nancy. Uruguay Police Agent Exposes US Advisors. NACLA'S Newsletter. New York: julio-agosto 1972, p. 20.

9 AGEE, Philip. Diario de la CIA. La 'Compañía' por dentro. Barcelona: Bruguera, 1979, p. 131 y 660.

10 SIEGEL, Daniel; y HACKEL Joy. El Salvador: la nueva visita de la contrainsurgencia. En: KLARE y KORNBLUH (coord.), op. cit., p. 149.

11 KLARE y STEIN, La exportación de instrumentos de represión, en: ibid., Armas y poder..., p. 172.

12 Ibid., p. 173.

13 LANGGUTH, op. cit., p. 307. 
consignaba el New York Times, 58. Su número variaba de país a país: uno en Chile o Jamaica, trece en Brasil. ${ }^{14}$ Aunque el informe anual del gobierno de Estados Unidos sobre asistencia al extranjero correspondiente al año fiscal 1968, indicaba la presencia de 91 consejeros de Seguridad Pública en el continente. ${ }^{15}$

Observa Michael McClintock que la asistencia del Programa de Seguridad Pública no desatendió enteramente el fortalecimiento policial convencional, pero los mayores esfuerzos se destinaron a la contrainsurgencia. "En consecuencia [el PSP] llegó a ser más conocido por su carácter de conducto del entrenamiento, la asistencia y el consejo operativo de la CIA a las Policías extranjeras, y por el hecho de vincular a Estados Unidos con los carceleros, torturadores y asesinos de los más represivos regímenes del 'mundo libre'". 16

\section{De la "Suiza de América" a la dictadura militar}

A mediados de los años sesenta, casi ningún indicio hacía presagiar que ocho años después Uruguay se hallaría sometido a una brutal dictadura. Para un observador internacional, el país parecía una excepción, una isla de bonanza en el mar de tempestades de América Latina. Los índices de salubridad, alfabetización, vivienda y empleo eran los más elevados del continente, y proverbial su estabilidad política. Hasta las conmociones de 1968, con sus secuelas de laceración política y social, nadie parecía percibir la precariedad del equilibrio institucional del país.

Nadie, con una excepción. Los analistas especializados en Uruguay del Departamento de Estado y la CIA, al estudiar la evolución de la política y la economía uruguayas, precisaron las líneas de tendencia del futuro inmediato y predijeron el advenimiento de una crisis grave, cuyos resultados aparecían peligrosamente inciertos para los intereses de Estados Unidos.

Por esta causa la embajada en Montevideo intensificó sus presiones para que el gobierno uruguayo aceptara la instalación de una misión de Seguridad Pública de la AID. Para lograr que esta aprobación llegara a buen puerto, fue necesario que los estadounidenses contaran con hombres receptivos a sus intereses,

14 The New York Times, 31.7.1970. «Two Kidnapped by Uruguayan Guerrillas».

15 KLARE, Michael. Operaciones policiales en América Latina. Marcha, Montevideo, 21.8.1970.

16 McCLINTOCK, op. cit., p. 5. 
ubicados en cargos clave: en primer término el ministro del Interior, Adolfo Tejera, el Jefe de Policía de Montevideo, coronel Ventura Rodríguez, y el subjefe coronel Carlos Martín, colaboradores políticos y de enlace de la estación montevideana de la CIA. ${ }^{17}$

Hasta fines de la década del cincuenta, el Parlamento uruguayo había opuesto resistencia a las ofertas de ayuda estadounidense, alegando razones de soberanía. En 1956 Estados Unidos había visto rechazadas sus propuestas de establecer programas de cooperación técnica general e industrial. Los acuerdos de asistencia eran aprobados por el Poder Ejecutivo, pero debían ser ratificados en la Cámara de Diputados por mayoría absoluta. Los legisladores de la fracción herrerista del Partido Nacional y de los partidos Socialista y Comunista desplegaban una tenaz oposición.

Dichos acuerdos, señalaba la embajada de Estados Unidos en Montevideo, eran absolutamente innecesarios para Uruguay, tanto desde el punto de vista técnico como del económico. No obstante, insistía en ellos por razones exclusivamente políticas, previendo las consecuencias de la declinación económica, que en un lapso muy breve seguramente sobrevendría.

El pluralismo político, la existencia de un gobierno colegiado y de una opinión pública sensible a la intromisión extranjera, explicaban, a juicio de la embajada, la resistencia opuesta a la ayuda norteamericana: “El gobierno es relativamente débil, con un Poder Ejecutivo descentralizado de nueve hombres y una oposición fuerte, activa y hostigante. Es un país relativamente adelantado, el tercero más alfabetizado en el hemisferio occidental, con un fuerte sentimiento nacionalista, un considerable nivel de autosatisfacción y algo de resentimiento ante la influencia o interferencia exterior. Existen muchos partidos políticos, o grupos dentro de los dos mayores partidos políticos, lo que vuelve dificultoso obtener apoyo o unidad frente a cuestiones específicas" ${ }^{18}$

Es bien conocido el destino que cupo a Uruguay desde 1959, cuando se sujetó a las normas del Fondo Monetario Internacional. La primera carta de intención fue firmada en 1960, reforzándose a partir de entonces los ya estrechos lazos de dependencia económica

17 AGEE, op. cit., p. 675, 682 y 684 .

18 Telegrama de Louis C. Nolan, director ICA Montevideo, a ICA Washington, 26.10.1956, "Long Term Country Plan and FY 1958 Program for Technical Assistance in Uruguay", NARA.RG286, box 110. El "General Agreement for Technical Assistance-Umbrella Agreement" había sido aprobado por el gobierno uruguayo y enviado a la Cámara de Diputados para su ratificación en marzo de 1956. 
del país. El 22 de marzo del mismo año, el Parlamento aceptó el resistido acuerdo general de cooperación técnica con Estados Unidos.

El 19 noviembre de 1963, el ministro del Interior Felipe Gil, también colaborador de la estación de la CIA de Montevideo, requirió oficialmente a la AID asistencia para el "mejoramiento de la fuerza policial". ${ }^{19}$ El convenio del gobierno uruguayo con la AID para establecer un Programa de Seguridad Pública de diez años de duración, financiado con fondos provenientes de ambos gobiernos, fue firmado en diciembre de 1964, en el marco del convenio general de $1960 .{ }^{20}$ Presidente del Consejo Nacional de Gobierno (CNG) era Luis Giannattasio. En Estados Unidos gobernaba Lyndon Johnson.

El propósito declarado del Programa era fortalecer a las fuerzas policiales en aspectos como administración, comunicaciones, investigaciones, patrullaje, entrenamiento, control de disturbios civiles y problemas relacionados. Otros eran los objetivos de fondo, que se detallan en los informes secretos o confidenciales que la misión de la AID o la embajada de Estados Unidos enviaban periódicamente desde Montevideo. El PSP era ante todo un programa de contrainsurgencia.

En un lapso de aproximadamente seis años, los consejeros de Seguridad Pública reorganizaron completamente a la Policía uruguaya y la capacitaron para la represión de los movimientos sociales y guerrilleros. Los núcleos donde invirtieron mayores esfuerzos y recursos fueron la Dirección Nacional de Información e Inteligencia y la Guardia Metropolitana.

Al igual que en otros países de América, Estados Unidos apostó primero al fortalecimiento de la Policía. A partir de 1971 (cuando el movimiento tupamaro había alcanzado su mayor expansión, y la izquierda se había unificado en un frente electoral) el énfasis y los recursos fueron desplazados hacia las Fuerzas Armadas. Los consejeros del PSP y los agregados del Grupo Militar de la embajada (MILGRP), prepararon gradualmente el terreno, impulsando desde 1965 la politización de los militares, al estimular su participación

19 AGEE, op. cit., p. 669. Memorando de Adolph Saenz, Montevideo, a Mr. Minotto, Washington [agosto 1965], "Uruguayan Police Force Survey", NARA.RG286, box 109.

20 Proyecto de renovación del PSP, suscrito por el Departamento de Estado/AID y el Ministerio del Interior de Uruguay, 11.9.1970, NARA.RG286, box 111. 
en la represión de los disturbios civiles. ${ }^{21}$ En principio, para consolidar una "segunda línea de defensa", la unión de Policía y Fuerzas Armadas en la lucha contrainsurgente. Este importante objetivo pudo concretarse en setiembre de 1971, cuando el gobierno de Jorge Pacheco encomendó a las Fuerzas Conjuntas la dirección de la campaña antisubversiva. ${ }^{22}$ En la eventualidad de que también esta línea cediera, siempre era posible para Estados Unidos recurrir a la "tercera línea de defensa": la ocupación del poder político por las Fuerzas Armadas mediante un golpe de Estado.

\section{Diez años de asistencia e intervención}

Entre 1965 y 1974 actuaron en Uruguay cuatro equipos del Programa de Seguridad Pública. Sus jefes fueron Adolph Saenz (enero de 1965 a julio de 1969), Daniel A. Mitrione (julio de 1969 a julio de 1970), Richard Martínez (interino entre agosto y setiembre de 1970), Roy Driggers (octubre de 1970 a junio de 1972) y Charles Guzmán (junio de 1972 a junio de 1974). Los consejeros a sus órdenes fueron sucesivamente César Bernal, Ronald Holko, William Cantrell, Julián Lindenauer, Lee Echols, Richard Biava, Richard Martínez, José Hinojosa y Jorge Matos. ${ }^{23}$

La OPS enviaba a los países latinos preferentemente instructores de origen hispanoamericano, para mimetizar su presencia y facilitar la comunicación y las relaciones de mando sobre la Policía local. ${ }^{24}$

La fuerza policial uruguaya a la que deberían capacitar y modernizar alcanzaba en 1966 los 6.064 efectivos en Montevideo y los 8.971 en el interior. En 1971, en el momento de aprobación de la Ley Orgánica policial, ascendía a 18.707 hombres. ${ }^{25}$

En 1972, con las Fuerzas Armadas sumadas a la Policía en la campaña contrainsurgente, esta última contaba en Montevideo con 10.150 efectivos, mientras los militares eran 11.000, en una población de 1.430.700 habitantes. En el interior, los policías eran

21 Véanse los informes mensuales AID Montevideo a AID Washington, "Public Safety Report" U-127, 1965-1969, NARA.RG286, box 110.

22 Así fue denominada la asociación de Policía y Fuerzas Armadas para la lucha contrainsurgente. La jefatura de la nueva institución recayó en las Fuerzas Armadas.

23 Datos obtenidos de los informes diarios y mensuales AID Montevideo a AID Washington 1965-1974, U-127, NARA.RG286.

24 KLARE, Michael; y STEIN Nancy. US Agents of Repression. NACLA'S Newsletter. New York: julio-agosto 1972, p. 23.

25 Memorando "Uruguayan Police Force Survey", DSP/AID Montevideo, 27.1.1966, NARA.RG286, box 109. OPS/AID, "Project Data Uruguay FY-1972. Public Safety Project" (1971), NARA.RG286, box 112. 
10.500 y los militares 16.000, para una población de 1.490 .300 personas. La relación en todo el país era, por lo tanto, de un policía o militar cada 61 habitantes. ${ }^{26}$

El equipo del PSP dependía administrativamente del representante de la AID en Montevideo. En este cargo se alternaron Franklin Stewart (hasta julio de 1969) y William Rhoads (desde 1969). Allen Claxton y Tom Stephens se desempeñaron como subjefes y directores interinos. Los embajadores de Estados Unidos fueron Wymberley R. Coerr (julio de 1962 a enero de 1965), Henry A. Hoyt (junio de 1965 a diciembre de 1967), Robert Sayre (agosto de 1968 a octubre de 1969), Charles W. Adair (noviembre de 1969 a setiembre de 1972), Frank V. Ortiz (interino, setiembre de 1972 a setiembre de 1973) y Ernest V. Siracusa (setiembre de 1973 a abril de 1977).

La misión de Seguridad Pública dependía de la Rama América Latina de la OPS e indirectamente del Buró América Latina de la AID. Entre los numerosos funcionarios que en Washington participaban en la toma de decisiones y evaluaban el trabajo de los consejeros policiales de Uruguay, se destacan los nombres de Peter Ellena, David R. Powell, Irving Tragen, Leonard Horwitz, Edward Marasciulo, David Cox, Robert McCarthy, Ted Brown, John Longan, Charles Brayshaw, James Todd, Jack Heller, Johnson Munroe, Harry Jacobson, Herman Myers y John Caldwell. ${ }^{27}$

Byron Engle seguía muy de cerca la actividad de sus consejeros en Uruguay. En mayo de 1971 visitó Montevideo para acordar con las autoridades uruguayas el apoyo de Estados Unidos, y en particular de la OPS, a la ofensiva antiguerrillera conjunta de Policía y Fuerzas Armadas, que se preparaba desde enero y sería oficializada en setiembre. ${ }^{28}$

Desde 1970 la figura más visible en la dirección de los asuntos uruguayos fue Peter Ellena, ascendido en 1971 a jefe de la Rama América Latina de la OPS. Al cumplirse un año de su estadía en Uruguay por el caso Mitrione, Ellena volvió a Montevideo. A fines de julio de 1971, se reunió con los máximos jerarcas del Ministerio

26 Carta de Charles Guzmán, jefe DSP Montevideo, a Lauren J. Goin, director OPS/ AID Washington, 9.5.1973. Anexo: “Uruguay. Security Forces Casualty Report CY 1972", NARA.RG286, box 112.

27 Datos extraídos de los documentos de OPS/AID citados en este trabajo.

28 Carta de Byron Engle, director OPS/AID, Washington, a William Rhoads, representante AID, Montevideo, 11.5.1971, NARA.RG286, box 112. AID Montevideo a AID Washington, "Public Safety Report May 1971" U-127, 25.6.1971, pp.11-16, NARA.RG286, box 110 . 
del Interior, presionó para que se dedicaran todos los recursos posibles a la resolución del caso, controló la marcha y potenciación del PSP y se trasladó a Brasil para interrogar a Aloysio Dias Gomide, recientemente liberado por el MLN. ${ }^{29}$

A mediados de 1972 Ellena dirigió un equipo de evaluación de la situación uruguaya. Junto a dos colaboradores de la OPS viajó a Montevideo para estudiar los resultados de la ofensiva antiguerrillera in situ. Elaboraron un estudio de más de 150 páginas, con conclusiones y recomendaciones, para presentar a la OPS. ${ }^{30}$

Los consejeros de Seguridad Pública, siguiendo las instrucciones de Washington, no sólo debían prestar asistencia, sino ejercer una "continuada influencia» para lograr los objetivos propuestos. ${ }^{31}$ Los sucesivos equipos del PSP que actuaron en Uruguay trabajaron en estrecha coordinación con el Grupo Militar de la embajada, dirigido desde 1967 por el coronel Lorenzo Caliendo, y con los servicios uruguayos de inteligencia policial y militar.

La Dirección Nacional de Información e Inteligencia (DNII) fue creada por impulso de los funcionarios de la CIA William Cantrell, consejero del PSP, y Juan Noriega, adscrito a la sección política de la embajada de Estados Unidos desde agosto de 1966 hasta noviembre de 1969. ${ }^{32}$ A partir de febrero de 1967 el núcleo de la CIA de Montevideo aportó fondos y entrenamiento para la puesta en marcha del nuevo organismo de seguridad. ${ }^{33}$

29 AID Montevideo a AID Washington, "Public Safety Report. July 1971" U-127, 17.8.1971, NARA.RG286, box 110.

30 OPS/AID, Washington, "Public Safety Evaluation-Uruguay, September 1972 (Draft)", secreto, NARA.RG286, box 109. El equipo estaba integrado por Ellena, jefe de la Rama América Latina de la OPS, Herman L. Myers y Bryan L. Quick. Permanecieron en Montevideo entre el 21 de setiembre y el 5 de octubre de 1972.

31 AID Washington, "Overseas Staffing Pattern Action Request (SPAR), Uruguay", 8.2.1966, p.2, NARA.RG286, box 110.

32 "Cantrell, William. Funcionario de operaciones de la CIA en Montevideo, adscrito bajo la tapadera de la Oficina de Seguridad Pública de la AID"; "Noriega, Juan. Funcionario de operaciones de la CIA en Managua y más tarde en Montevideo". Antes de ser trasladado a Uruguay, Noriega era responsable del adiestramiento de los guardaespaldas de Anastasio Somoza y su familia. AGEE, op. cit., p. 521, 536537, 660 y 678. La creación de la DNII "bajo la directa guía" de Cantrell en: AID Montevideo a AID Washington, "Terrorist Activity in Uruguay. December 1966 and January 1967", 10.2.1967, NARA.RG286, box 114.

33 HEVIA COSCULLUELA, Manuel. Pasaporte 11333. Ocho años con la CIA. La Habana: Editorial Ciencias Sociales, 1978, p. 225-242. El autor fue agente doble de inteligencia al servicio de la CIA y de la seguridad cubana. Actuó en Uruguay entre 1964 y 1970, bajo la dirección de Cantrell, Noriega y Richard Martínez. Véase también: "Declaraciones al MLN de Nelson Bardesio" (1972), en Embajada de EEUU en Montevideo a secretario de Estado William Rogers, Montevideo 00876, 17.4.1972, NARA.RG59, box 2662. 
A través de la DNII, la CIA pudo desarrollar con mayor libertad su red clandestina en los servicios de seguridad y orientar la política contrainsurgente. Como en otros países de América Latina, el PSP serviría de cobertura a las operaciones conjuntas de la Agencia con la Policía local y facilitaría la dirección encubierta de la represión política y de los servicios de inteligencia.

Los sucesivos jefes de la división uruguaya del PSP y sus consejeros de investigaciones, supervisaron y orientaron paso a paso el trabajo de la DNII hasta junio de 1974, cuando el Programa fue clausurado: desde las actividades de inteligencia (incluyendo la infiltración en el Partido Comunista, la central sindical y las organizaciones armadas) hasta el entrenamiento y las operaciones contra la guerrilla en todas sus fases, en Montevideo y el interior del país.

Desde comienzos de los años sesenta la CIA había montado su aparato paralelo dentro del servicio secreto policial, el Departamento de Inteligencia y Enlace. ${ }^{34}$ También estableció un vínculo con la por entonces incipiente inteligencia militar. Una red de informantes, agentes y colaboradores, encabezada por jerarcas policiales y militares, realizaba acciones ilegales, espionaje (también contra su propio gobierno) y manipulación política por cuenta de la CIA, que remuneraba con prodigalidad sus servicios. ${ }^{35}$

En una reciente entrevista el ex comisario Alejandro Otero observaba al respecto: "Inteligencia y Enlace respondía siempre a lo que eran las necesidades de los servicios de inteligencia americanos. Cuando yo me hago cargo, actúo con total independencia y queda esclarecido que toda la información que yo obtenía, toda, yo la proporcionaba a esos servicios, porque así estaba ordenado". ${ }^{36}$

Lo cierto es que la CIA desplegaba una intensa actividad en Uruguay antes de la crisis de 1968; actividad que se incrementó en los años sucesivos. A tal punto que en abril de 1973, la embajada se

34 En 1968, al ser incluido en la estructura de la DNII, Inteligencia y Enlace pasó a denominarse Departamento 4.

35 Entre los numerosos agentes y colaboradores de la CIA que Agee individualiza se hallaban el jefe de la Guardia Republicana teniente coronel Mario Barbé, los sucesivos jefes de Policía de Montevideo coroneles Mario Aguerrondo, Rogelio Ubach y Ventura Rodríguez, el subjefe de Policía coronel Carlos Martín, el comisario Alejandro Otero, el subcomisario Pablo Fontana, el inspector Antonio Pírez Castagnet, los jefes de la Guardia Metropolitana coronel Roberto Ramírez y teniente coronel Amaury Prantl, el subjefe de Investigaciones Juan José Braga, el jefe de inteligencia militar coronel Carvajal y el teniente coronel Zipitría. AGEE, op. cit., p. 653-687.

36 Alejandro Otero, entrevista de la autora, Montevideo, 2002. 
lamentaba ante Washington por la gran proporción de funcionarios con cobertura oficial (aproximadamente 150) destinados a "recoger y procesar abierta o encubiertamente la inteligencia obtenida". Sin contar los que revistaban con cobertura no oficial. Una presencia que respondía a "requerimientos impuestos desde Washington". El Country Team comprendía, con todo, que la "mayor inhibición para reducir el staff de la misión" eran los objetivos perseguidos por Estados Unidos, particularmente "en los campos de inteligencia y militar". ${ }^{37}$ En 1972 el personal adscrito a la embajada alcanzaba los 363 funcionarios. ${ }^{38}$

El golpe de Estado de 1973 - señalaban - permitiría por fin reducir el extraordinario número de funcionarios dedicados a tareas de inteligencia. "Uruguay, en relación a los intereses de Estados Unidos en este hemisferio, ocupa una posición modesta y secundaria. El comercio, las inversiones y los intereses estratégicos de Estados Unidos son verdaderamente pequeños. La comunidad norteamericana es también muy pequeña. A pesar de estos factores, nuestra presencia oficial es desproporcionadamente numerosa. El Country Team piensa que nuestra alta visibilidad es un factor que complica el desarrollo de relaciones realistas". Entre otros inconvenientes, observaban, había facilitado a la izquierda marxista nuevos pretextos para enfatizar la intromisión en los asuntos internos del "ogro imperialista". 39

$$
* * *
$$

La racionalización de la estructura policial, con la centralización y coordinación de sus divisiones a nivel nacional y la creación de nuevos organismos, se fue cumpliendo a impulso de los consejeros de Seguridad Pública. La Ley Orgánica policial, aprobada en mayo de 1971, fue una de sus más ambiciosas metas. Otros objetivos alcanzados fueron la creación de un registro nacional de la

37 Embajada de EEUU en Montevideo a Departamento de Estado, "Country Analysis and Strategy Paper-Uruguay, 1974-1975 (CASP)", secreto, 4.4.1973, p.1, NARA.RG59, box 2662.

38 Discriminados como sigue: 208 por el Departamento de Estado (incluyendo 10 marines, 69 guardias y 8 choferes, entre los cuales también podían enmascararse funcionarios de inteligencia), 44 por la AID, 58 por el USIS, 38 por el Departamento de Defensa, 17 por los Cuerpos de Paz, 1 por el FAS. Para 1973 y 1974 se planeaba acrecentar el personal a 385 y 386 personas respectivamente, ibid., p.1, 8, 9, Anexo A, Cuadro 2, p. 77. "Country Analysis and Strategy Paper-Uruguay, 1973-1974 (CASP)", secreto, 23.2.1972, Anexo A, Cuadro 3, p.60, NARA.RG59, box 2662.

39 Embajada de EEUU en Montevideo a Departamento de Estado, "Country Analysis and Strategy Paper-Uruguay, 1974-1975 (CASP)", secreto, 4.4.1973, p. 1 y 9. NARA.RG59, box 2662. 
identidad de los habitantes, la centralización de la información sobre las personas, el control de fronteras, la construcción de una moderna Escuela de Policía (para la que aportaron sostén financiero), el desplazamiento de las prisiones a la órbita del Ministerio del Interior y el establecimiento de cárceles de máxima seguridad.

La prensa de izquierda de todas las corrientes y la de algunos sectores del Partido Nacional, expresó tempranamente su preocupación por la presencia de asesores norteamericanos y vinculó a su asistencia el vertiginoso aumento de capacidad represiva. Pero manejaba una información muy general y a menudo equivocaba los nombres de personas y de organismos a los que se adscribían. Desconocía también la complejidad de sus funciones. En ocasiones, las denuncias de injerencia otorgaban la misma jerarquía al supuesto espionaje realizado por los Cuerpos de Paz, los mormones y otros miembros de iglesias reformadas, que a la presencia de instructores estadounidenses en las fuerzas represivas. ${ }^{40}$

Durante diez años fue un ir y venir de técnicos estadounidenses y de otras nacionalidades para perfeccionar aspectos de la labor policial, remunerados con fondos del Programa. Los informes elaborados por estos visitantes, revelan que la dirección de la Policía uruguaya en sus actividades contrainsurgentes no residía en el Ministerio del Interior, sino en la OPS de Washington y en la embajada de Estados Unidos.

El rechazo a la presencia norteamericana en vastos sectores de opinión, indujo a los sucesivos embajadores a reducir al mínimo el número de consejeros y recurrir a estadías cortas de expertos o instructores. Periódicamente llegaban a Uruguay funcionarios de alto rango de la OPS o del Pentágono para supervisar el trabajo y atender los requerimientos de la asistencia. Se reunían con las máximas autoridades de los Ministerios de Interior y Defensa; inspeccionaban instalaciones militares o policiales uruguayas y se informaban en directo de las operaciones y actividades de inteligencia, procedimientos que constituían una franca violación de la legislación uruguaya. Cabe señalar que sus estadías se efectuaban ante la completa ignorancia del Parlamento, la prensa y la opinión pública. Los periodistas, intelectuales y parlamentarios de la oposición, centraban su atención en las intromisiones en política interna de los embajadores norteamericanos cuando

40 Un artículo emblemático es el publicado el 1.10.1969 por El Popular: "Un nuevo edificio para espiarnos mejor". El periodista colocaba en un mismo plano la presencia de estadounidenses en las Fuerzas Armadas y en las plazas de deportes. 
pronunciaban discursos. En el caso de Charles Adair, por ejemplo, fueron criticadas en 1971 "las múltiples ocasiones en que el actual embajador ha vulnerado el principio de no intervención en nuestros asuntos internos con sus declaraciones". ${ }^{41}$

Uruguay fue durante 1971 y 1972 un campo de experimentación para las políticas contrainsurgentes aplicadas posteriormente por Estados Unidos en otros países. "Los agudos problemas de seguridad interna en un pequeño país como Uruguay, proporcionan la oportunidad de experimentar la factibilidad de un esfuerzo completamente coordinado y de evaluar nuevas técnicas operativas implementadas a escala limitada por las agencias de Estados Unidos involucradas", concluyeron dos enviados del Departamento de Estado y Defensa que inspeccionaron los avances de la ofensiva antiguerrillera de las Fuerzas Conjuntas a mediados de $1972 .{ }^{42}$

\section{Armas, equipos y adiestramiento contrainsurgente}

Mientras el país se debatía en una grave crisis económica, los gobiernos de los Partidos Nacional y Colorado importaban armas letales y todo tipo de tecnología represiva para enfrentar a la oposición. Los instrumentos represivos utilizados desde 1965 contra los movimientos sociales y guerrilleros fueron proporcionados en su casi totalidad por el PSP y el MAP.

Aunque la embajada cuidaba celosamente que la posición de privilegio de Estados Unidos en ventas de equipos, automóviles y armamento no fuera suplantada por otros países, a fines de 1969 el embajador Charles Adair decidió que las armas y municiones fueran compradas por intermedio de Argentina o Brasil, para acallar las denuncias de la izquierda, que acusaba a Estados Unidos de proporcionar al gobierno instrumentos de muerte. ${ }^{43}$

41 REAL DE AZÚA, Carlos. Partidos, política y poder en el Uruguay. 1971, coyuntura y pronóstico. Montevideo: Universidad de la República, 1988, p. 146, n. 61.

42 Memorando de los inspectores Julius L. Okun y Donald B. Kraft al Secretario de Defensa, Melvin Laird, y al Administrador de la AID, John A. Hannah: "Internal Security Assistance in Uruguay", 29.6.1972, NARA.RG286, box 111.

43 En 1968 se intensificaron las presiones de la embajada y los consejeros del PSP amenazando la suspensión de la ayuda estadounidense, ante el proyecto del Ministerio del Interior de comprar vehículos Volkswagen a Alemania y armamento a Checoslovaquia: AID Montevideo a AID Washington, "Public Safety Monthly Report. May 1968" U-127, 15.6.1968, p.6, NARA.RG286, box 110. La compra de armamento a través de terceros países en: Embajada de EEUU en Montevideo a secretario de Estado, Montevideo 1109, 11.4.1973, NARA.RG59, box 2662. En esta comunicación se recordaba a Washington que Adair había dispuesto dicha política a fines de 1969, con el acuerdo del Departamento de Estado, ante las frecuentes denuncias de los "marxistas y otros elementos de la oposición" sobre las armas y municiones que seguían llegando de Estados Unidos "para matar otros uruguayos". 
Entre diciembre de 1966 y junio de 1971, la OPS envió a Uruguay partidas de armamento ligero para uso policial: revólveres, escopetas, rifles y municiones. En agosto de 1968 despachó por avión 50 escopetas antimotines, con las que efectivos de la Guardia Metropolitana asesinaron el 20 de setiembre a los estudiantes Hugo de los Santos y Susana Pintos. La orden de disparar contra los manifestantes fue impartida por el jefe de la Metropolitana, coronel Alfredo Rivero, que comandaba los efectivos policiales y había sido instruido en "control de disturbios" por el PSP. En el informe mensual enviado a Washington, los estadounidenses relativizaron las muertes, precisando que las víctimas eran integrantes de la Juventud Comunista. ${ }^{44}$ En el Parlamento se inquirió, infructuosamente, acerca del origen de las nuevas armas. El presidente Pacheco y sus ministros inauguraron procedimientos destinados a perdurar en los años sucesivos: la negación de los crímenes de Estado, el ocultamiento de la verdad y la desautorización del Parlamento. ${ }^{45}$

Desde julio de 1966 hasta mayo de 1973, la OPS suministró ingentes partidas de equipos y municiones químicas para el control de tumultos. Los envíos más consistentes fueron efectuados durante el auge de conflictos y movilizaciones sociales, entre 1968 y 1969. Escopetas y revólveres de gas "Federal", miles de granadas, proyectiles y cartuchos de gas lacrimógeno CN y CS (destinadas a la Guardia Metropolitana); quintales de gas lacrimógeno en bidones, dispensadores en aerosol del mismo gas, Maza Química MKIV y MKII, "Pepper Fog" y "Niebla irritante". También proporcionó granadas y proyectiles de gas de dispersión detonante. Cientos de manifestantes resultaron heridos, algunos de gravedad, por su explosión cercana o impacto directo. ${ }^{46}$

44 ID Montevideo a AID Washington, «Public Safety Report September 1968» U-127, 16.10.1968, NARA.RG286, box 110. El consejero estadounidense César Bernal había entrenado en agosto a los policías de la Metropolitana en el uso de las nuevas armas. AID Montevideo a AID Washington, "Public Safety Monthly Report August 1968" U-127, 28.9.1968, p.3a, NARA.RG286, box 110.

45 Hasta el 22 de setiembre de 1968 el ministro de Cultura, Federico García Capurro, negó terminantemente que la Metropolitana hubiera empleado armas de ese tipo. Durante la interpelación parlamentaria, en octubre de 1968, el ministro del Interior Eduardo Jiménez de Aréchaga, de filiación colorada, debió admitir su uso, pero aseguró que habían sido compradas por el anterior gobierno del Partido Nacional. Fue desmentido por el senador Felipe Gil. Pero la verdad sobre el origen de este armamento nunca llegó a conocerse. VARELA, Gonzalo. El movimiento estudiantil de 1968. El IAVA, una recapitulación personal. Montevideo: Trilce, 2002, p. 114-115.

46 Departamento de Estado, OPS/AID, "US Public Safety Project. Fiscal Years 19641973", Anexo: “Equipos de control de tumultos", NARA.RG286, box 116. 
El modelo de las cachiporras de madera de 26 pulgadas fue introducido en 1965 por el primer consejero, Adolph Saenz. Fueron adoptadas de inmediato y comenzaron a fabricarse en serie en Uruguay. Una prueba inaugural de su eficacia fue supervisada por Saenz en el invierno de 1965, durante los apaleos de manifestantes y huelguistas. ${ }^{47}$ En 1968 el aumento de la demanda obligó a los estadounidenses a incentivar su producción. ${ }^{48}$

Un aspecto particular de la asistencia OPS en América Latina fue el área de las radiocomunicaciones policiales. Permitía el intercambio y la coordinación regional de información sobre la «amenaza subversiva» y sobre aspectos de competencia policial como la lucha al tráfico de drogas. La OPS proveía los equipos y la asistencia técnica. En 1965 los consejeros de Seguridad Pública comenzaron a instalar en Uruguay una moderna red de radiocomunicaciones que fue completada en 1971. ${ }^{49}$ En 1966 la estación uruguaya de la CIA comenzó a interceptar las comunicaciones de la red. ${ }^{50}$ Cabe suponer que cuando conectó a la presidencia de la República, las comunicaciones de la máxima autoridad del Estado también fueron interceptadas por la CIA. El costo de los equipos de comunicaciones, del adiestramiento para su uso y reparación, de los repuestos y accesorios, fue compartido entre la OPS y el gobierno uruguayo. El network instalado en estos años por el PSP permitió que Uruguay participara en la red de comunicaciones "Condortel", que conectó a los servicios secretos activos en la Operación Cóndor. ${ }^{51}$

El Programa distribuyó vehículos Ford y Chevrolet a las unidades policiales de todo el país. También financió la adquisición

47 AID Montevideo a AID Washington, "Monthly Report on the Public Safety Program for Montevideo. July 1965" U-127, 13.8.1965, p.4, NARA.RG286, box 110.

48 AID Montevideo a AID Washington, "Public Safety Monthly Report. June 1968" U127, 13.7.1968, p. 4, NARA.RG286, box 110.

49 Conectó a los Ministerios de Interior y Defensa, la Casa de Gobierno, las jefaturas de Policía de Montevideo e interior, todas las seccionales policiales del país, las Guardias Metropolitana y Republicana, los aeropuertos de Carrasco y Melilla, la embajada de Estados Unidos y la residencia del embajador, las Inspecciones del Ejército, la Fuerza Aérea y la Marina, el Centro de Operaciones y la Oficina de Asistencia Técnica del PSP en la Jefatura de Policía de Montevideo, Radio Patrulla y la Casa Militar. Departamento de Estado, OPS/AID, "US Public Safety Project. Fiscal Years 1964-1973", Anexo: “Equipos de Comunicación", NARA.RG286, box 116.

50 "[Adolph] Saenz me facilitó las frecuencias y con nuestros propios receptores podremos controlar las frecuencias de la Policía", escribía Agee en mayo de 1966. AGEE, op. cit., p. 521.

51 McSHERRY, Patrice Death Squads as Parallel Forces: Uruguay, Operation Condor, and the United States. Journal of Third World Studies, New York: vol. XXIV, $\mathrm{n}^{\circ} 1$, 2007, p. 9. 
de ómnibus, jeeps, camiones, camionetas, patrulleros, ambulancias, trailers, motos y repuestos. ${ }^{52}$

Los equipos y municiones utilizados en los cursos regionales y en el Instituto de Enseñanza Profesional fueron proporcionados desde 1966 por la OPS. El laboratorio técnico policial fue completamente modernizado y sus funcionarios recibieron cursos en Estados Unidos y países de la región. Se enviaron avanzados equipos de investigación y laboratorio. La OPS proveyó a la Policía de un sinnúmero de otros implementos necesarios para su actividad. ${ }^{53}$

La compra o donación de armamento y equipos estadounidenses aseguraba la dependencia logística de las fuerzas represivas. Mantenimiento, repuestos y técnicos debían ser suministrados por el país proveedor.

Hasta 1973 la OPS invirtió, en materiales y adiestramiento, 2.131.258 dólares. Utilizando la línea de crédito abierta por un fondo fiduciario de la AID, el gobierno uruguayo contribuyó con 1.237.637 dólares, de los cuales 887.987 fueron destinados a la adquisición de vehículos, 273.214 a equipos de comunicaciones y el resto a otros rubros. ${ }^{54}$

$$
\text { *** }
$$

Los oficiales con mayores perspectivas de ascenso o quienes ya ocupaban puestos de mando fueron seleccionados por los consejeros para recibir cursos en Estados Unidos. Se adiestraban o perfeccionaban en investigaciones, patrullaje, comunicaciones, inteligencia, control de disturbios y administración policial. Desde 1969 los cursos incluyeron materias como seguridad de aerolíneas, bombas, explosivos y protección de dignatarios. ${ }^{55}$

El adiestramiento se realizaba fundamentalmente en la IPA, pero también en la Academia Nacional del FBI, la Escuela Internacional de Servicios de la Policía (IPS), la Oficina de Control de Fronteras, los Departamentos de Prisiones policiales de varios

52 El costo unitario en 1969-1970 de un Ford Falcon era de 2.767 dólares, de un jeep 2.267, de un Chevy Nova 2.660; de un autobús 7.200. Departamento de Estado, OPS/ AID, "US Public Safety Project. Fiscal Years 1964-1973", Anexo: "Vehículos", NARA.RG286, box 116.

53 Ibid. Anexos: "Equipos de Adiestramiento", "Equipos de Investigación y Laboratorio", "Equipos de táctica», «Equipos sobrantes y diversos".

54 Estas cifras fueron tomadas de ibíd.; y de AID/Public Safety Program Uruguay, "Financial Status Report 1964-1973", NARA.RG286, box 116.

55 Departamento de Estado, OPS/AID Washington, "Terrorism, a National and International Problem. The Office of Public Safety Seminar", 15.9.1973, NARA.RG286, box 10. 
Estados, unidades del Ejército y del Departamento de Interior y escuelas de radiocomunicación policial. Un número menor de oficiales uruguayos fue enviado por el PSP a recibir adiestramiento a la Policía Federal argentina, a la Escuela de Carabineros de Chile y a Venezuela. ${ }^{56}$

La IPA se dividía en tres departamentos: Administración Policial (que adiestraba en organización, comando, vinculaciones a nivel de jefatura y relaciones públicas); Operaciones Policiales (en criminalística, comunicaciones, controles fronterizos e inteligencia) y Seguridad Interna (en control de motines, municiones químicas y medidas antiterroristas). Algunos policías becados a la IPA fueron enviados al Centro Militar Especial John F. Kennedy de Fort Bragg, en Carolina del Norte, donde recibían un curso en operaciones antiinsurreccionales y de apoyo policial a las operaciones militares no convencionales. ${ }^{57}$

Un documento de la OPS consigna un total de 169 policías uruguayos instruidos con fondos del PSP en Estados Unidos entre 1966 y 1974, detallando las fechas y el tipo de curso recibido. ${ }^{58}$

Los graduados de la IPA $\mathrm{u}$ otras escuelas ocuparon progresivamente los puestos de mando de la Policía. Los más jóvenes, a su regreso a Uruguay fueron encargados del entrenamiento o tareas de instrucción. En 1972, de los 39 oficiales que habían recibido adiestramiento en Estados Unidos a nivel ejecutivo en supervisión, dirección e instrucción, 37 ocupaban las máximas

56 Datos extraídos de los sucesivos «Public Safety Report» U-127, 1965-1971, NARA.RG286; y de FERNÁNDEZ, Wilson. El gran culpable. La responsabilidad de los EEUU en el proceso militar uruguayo. Montevideo: Atenea, 1986, p. 224 y ss. La precedente Academia Interamericana de Policía (IAPA), establecida por el presidente Kennedy en 1962 en Fort Davis, en la Zona del Canal de Panamá, fue trasladada en 1964 a Washington con el nombre de Academia Internacional de Policía (IPA). Fundada por la AID, estaba en realidad controlada por la CIA. La IPS, ubicada en Washington, era una escuela de adiestramiento de la CIA con cobertura comercial. AGEE, op. cit., p. 653 y 666.

57 KLARE, Operaciones policiales en América Latina, op. cit. El autor toma estos datos de la publicación mensual de la Academia, IPA Review, enero de 1967, p. 11 y 12. Un documento de la OPS de 1973, citado por McCLINTOCK, op. cit., p. 5, n. 64, indica que los policías becados a la IPA recibían en Fort Bragg un curso de 22 horas, con materias como "Operaciones tácticas de contraguerrilla", "Metodología subversiva insurgente" y "Control de población y de recursos".

58 Reproducido en FERNÁNDEZ, op. cit., p. 224 y ss. La lista es incompleta; en ella no figuran, entre otros, Alejandro Otero, Antonio Silveira Regalado, Ramiro Fernández Regueiro y Gervasio Somma Tabeira, cuyos entrenamientos son mencionados en: Embajada de EEUU en Montevideo a secretario de Estado Dean Rusk, Montevideo 3316, "Public Safety Training. Significant Achievements of Public Safety Participants", 16.6.1968, NARA.RG286, box 113. 
jerarquías policiales. ${ }^{59}$ No todos los oficiales seleccionados por los consejeros para recibir los cursos en Estados Unidos se comprometieron en violaciones a los derechos humanos. No obstante, algunos fueron connotados torturadores, integrantes del escuadrón de la muerte o ejecutores de secuestros, asesinatos y desapariciones en el marco de la Operación Cóndor. Entre ellos, Héctor Morán Charquero, Juan María Lucas, Víctor Olivio Castiglioni, Hugo Campos Hermida y Ricardo Medina Blanco. ${ }^{60}$ De hecho, la tortura se volvió desde 1968 el procedimiento habitual en los interrogatorios policiales a los opositores políticos: guerrilleros, dirigentes sindicales, estudiantes o trabajadores detenidos en manifestaciones y huelgas.

Los cursos en Washington cumplían una finalidad política, además de profesional: encauzaban las opiniones y sentimientos de los becados, generando una corriente de simpatía hacia los intereses y objetivos de Estados Unidos. El adiestramiento incluía lecturas y discusiones de material ideológico anticomunista. Durante la estadía se realizaban viajes turísticos y visitas guiadas a lugares de interés histórico o político. Se les hospedaba en hoteles lujosos y se les agasajaba con fiestas y paseos. En el transcurso del adiestramiento se fomentaba el contacto personal y el estrechamiento de lazos amistosos con los instructores y los compañeros latinoamericanos. ${ }^{61}$

En Uruguay, los consejeros del PSP impartían directamente algunos cursos básicos similares a los de Estados Unidos, supervisaban otros y distribuían material de adoctrinamiento. $L a$ primera línea de defensa, un filme de culto del PSP, era proyectado desde 1965 a jerarcas policiales y militares, comisarios y subalter-

59 Carta de Charles Guzmán, jefe DSP / AID, Montevideo, a Byron Engle, director OPS / AID, Washington, 26.2.1973, Anexo: "Uruguayan Police Achievements", p.3, NARA.RG286, box 111. Informe de la DSP, Montevideo, a OPS/AID, Washington, "Montevideo Police Organization", [mayo 1971], NARA.RG286, box 115.

60 Cámara de Senadores de la República Oriental del Uruguay, Actas de la Comisión Especial Investigadora sobre violaciones de los derechos humanos y comisión de actos de torturas a detenidos y regímenes de detención vejatorios a la dignidad humana. Montevideo, diciembre de 1969-mayo de 1970 (mimeo interno). SERPAJ (Servicio Paz y JusticiaUruguay). Uruguay nunca más. Informe sobre la violación a los derechos humanos (19721985). Montevideo: SERPAJ, 1989. Madres y Familiares de Uruguayos Detenidos Desaparecidos. A todos ellos. Montevideo: Caligráficos, 2005.

61 "Documentos Miguel A.Benítez" en COSTA GAVRAS, Constantin; y SOLINAS, Franco. State of Siege. New York: Ballantine Books, 1973, p. 161-180.; KLARE, Michael. La logística de la represión: el apoyo norteamericano al autoritarismo en el extranjero, en: KLARE y STEIN, Armas y poder..., p. 11-25. 
nos. Según los informes de los consejeros, despertaba interés, emoción y sentimientos de cohesión entre los asistentes. ${ }^{62}$

Los efectivos policiales y militares uruguayos que participaban en los cursos efectuaban periódicamente demostraciones de sus habilidades ante los funcionarios de la embajada. El entrenamiento en el uso de armas y equipos antimotines de policías y militares, se realizaba ante la presencia y supervisión de los instructores del PSP y del MILGRP. ${ }^{63}$

El adiestramiento de la Policía del interior mantuvo un ritmo sostenido desde 1965, pero se aceleró a comienzos de 1971, cuando se volvió segura una pronta extensión de la actividad guerrillera a las zonas rurales y poblaciones del interior. Equipos móviles de instrucción, dirigidos por oficiales adiestrados en la IPA, se desplazaron a lo largo y ancho del país, proporcionando cursos intensivos en los 18 departamentos. Los consejeros de Seguridad Pública viajaron frecuentemente al interior para inspeccionar los resultados.

También supervisaron, junto a los Jefes de Policía de varios departamentos, el desarrollo de secciones de inteligencia que establecieran una radiografía precisa de las ideas y filiaciones políticas de la población del área. Cientos de personas fueron vigiladas discretamente, se registraron sus movimientos de salida de la ciudad de residencia, sus transacciones inmobiliarias y las de sus familiares. ${ }^{64}$ Esta información permitió que las Fuerzas Armadas iniciaran en setiembre de 1971, con apreciables resultados, la represión contrainsurgente.

En los primeros meses de 1970, Mitrione comenzó a enviar a Estados Unidos jóvenes policías de la DNII para que recibieran un nuevo curso de la IPA, el "Terrorist Activities Investigations Course" (TIC). ${ }^{65}$ Adiestraba a los reclutas en acciones terroristas, como la manufactura y colocación de bombas de construcción artesanal y artefactos incendiarios, la voladura con explosivos de

62 Las reacciones ante la primera proyección del filme en: AID Montevideo a AID Washington, "Monthly Report on the Public Safety Program April 1965" U-127. Cablegrama de Adolph Saenz, jefe DSP Montevideo, a AID Washington, U-127, 14.3.1966, p.4; NARA.RG286, box 110 .

63 Al término de los cursos o ejercicios, se enviaban a Washington informes y fotografías de los participantes uruguayos y los funcionarios estadounidenses, conservados en el fondo RG286 del NARA.

64 Véase por ejemplo: AID Montevideo a AID Washington, "Public Safety Monthly Report May 1971" U-127, 25.6.1971, NARA.RG286, box 110.

65 Denominado en algunos documentos de difusión pública "Technical Investigations Course" (TIC). 
vehículos en marcha, viviendas particulares, edificios públicos, torres de electricidad y depósitos de combustible. También entrenaba en técnicas para cometer asesinatos con arma blanca sin dejar evidencia. La parte teórica se desarrollaba en la IPA y las prácticas de campo en la Academia de Control de Fronteras, ubicada en Los Fresnos, Texas. Aunque los cursos eran financiados por la AID, los instructores eran proporcionados por la CIA. En 1973, 16 policías uruguayos habían recibido esta capacitación. ${ }^{66}$ Entre ellos un tupamaro, el suboficial de la DNII Miguel Ángel Benítez Segovia. ${ }^{67}$ En setiembre de 1973 se habían completado siete cursos TIC de ocho semanas de duración, impartidos en inglés o español a 140 participantes del Tercer Mundo. ${ }^{68}$

\section{Conclusiones}

A través de los programas de asistencia policial y militar, Policía y Fuerzas Armadas recibieron un flujo de recursos destinados a reorientar su función hacia cometidos de "seguridad interna" y "defensa nacional". Es decir, hacia la represión de quienes pudieran establecer en el mediano y corto plazo "un gobierno hostil a los Estados Unidos". ${ }^{69}$ Ya fuera de carácter revolucionario como reformista y nacionalista. El objetivo era evitar que en Uruguay triunfara un Fidel Castro, pero también un Joâo Goulart o un Salvador Allende.

En este período Uruguay ingresó de lleno en las lógicas de la guerra fría y la división del mundo en áreas de influencia. El presidente Pacheco y su sucesor Juan María Bordaberry pudieron privilegiar el uso de la fuerza para enfrentar los conflictos sociales y la ofensiva guerrillera, desestimando las posibilidades de diálogo

66 KLARE y STEIN, Terrorismo policíaco en Latinoamérica, en: ibid., Armas y poder..., p. 68-81.

67 Según se consigna en una carta de Johnson F. Munroe, OPS/AID Washington, a Lee Echols, OPS/TD Washington, 25.4.1972, NARA.RG286, box 113, Benítez recibió el segundo curso TIC. A su regreso a Montevideo continuó sus actividades policiales de inteligencia (era suboficial de la DNII, bajo el mando del comisario Juan María Lucas). En 1971 y 1972 trabajó como guardia nocturno de la residencia de los marines asignados a la custodia de la embajada de Estados Unidos. Su pertenencia al MLN fue descubierta en abril de 1972. Estuvo preso desde esa fecha hasta el término de la dictadura.

68 Departamento de Estado, OPS/AID Washington, «Terrorism, a National and International Problem. The Office of Public Safety Seminar», 15.9.1973, «AID/OPS Training», por John A. Lindquist, p. 27, NARA.RG286, box 10.

69 Embajada de EEUU en Montevideo a Departamento de Estado, «Country Analysis and Strategy Paper-Uruguay, 1973-1974», secreto, 23.2.1972, NARA.RG59, box 2662. 
y la adopción de una política reformista, también porque Estados Unidos, en un sostenido y discreto trabajo iniciado en 1965, les había proporcionado el instrumento idóneo.

El Programa de Asistencia Militar se volvió desde 1971 la principal herramienta de Estados Unidos para influir en las decisiones políticas y el futuro del país. Las Fuerzas Armadas uruguayas se subordinaron a la visión estadounidense de que todo aquello que afectara la seguridad nacional de Estados Unidos, constituía una grave amenaza para Uruguay y el conjunto de las naciones latinoamericanas. Pese a su tradicional apoliticismo, los militares terminaron adoptando, al igual que sus camaradas de la región, la conceptualización de esta política expresada en la doctrina de la seguridad nacional.

La ayuda estadounidense no proveyó a Uruguay de equipamiento militar avanzado para una guerra convencional, sino que invirtió en armas, equipos, asistencia técnica, apoyo logístico y entrenamiento para la represión interna. En un primer momento para sofocar violentamente los conflictos sociales, más tarde para aniquilar a la guerrilla. Pero cuando estos objetivos parecían alcanzados, se perfiló en el horizonte una nueva amenaza para los intereses de Estados Unidos en Uruguay. Una fuerza política de reciente formación, el Frente Amplio, puso fin al tradicional bipartidismo y se propuso disputar la hegemonía de los partidos Nacional y Colorado. Pese a la concluyente victoria de estos partidos en las urnas, en noviembre de 1971, en Washington se consideró muy probable la victoria de la coalición de izquierdas en las elecciones previstas para 1976.

Por esta razón, a comienzos de 1972 la embajada proponía que Estados Unidos concentrara sus esfuerzos y orientara su injerencia en Uruguay - mediante acciones públicas o encubiertas - diseñando programas "que deberían apoyar con decisión durante los próximos cinco años las fuerzas democráticas que en Uruguay se oponen a la coalición Frente Amplio, dominada por los marxistas". El primer objetivo de Estados Unidos y de su embajada se volvió impedir que la izquierda triunfara en las urnas en 1976, reeditando el caso chileno: "El alto nivel de preocupación por nuestros intereses deriva del serio revés que sufrirían nuestros esfuerzos por impedir la expansión de la influencia comunista en este hemisferio si ocurriera una victoria marxista en Uruguay. Esta victoria se volcará contra la política de Estados Unidos de promover paz y desarrollo, mientras el gobierno uruguayo liderado por los mar- 
xistas conducirá a la violencia y a programas radicales adversos para nuestros intereses. Planteará una seria amenaza a Brasil y Argentina y además tendrá una influencia desestabilizadora en esta área. Por estos factores, el gobierno de Estados Unidos debe emprender un alto nivel de esfuerzos en el período planificado".$^{70}$

Para neutralizar la fuerza potencial del Frente Amplio se volvió insuficiente el solo recurso a la represión. Era necesario ensayar otras soluciones. No casualmente, el golpe de Estado de 1973 resolvió por muchos años las inquietudes de Estados Unidos y sus aliados vernáculos. ${ }^{71}$

$$
* * *
$$

La actitud de la Policía y de las Fuerzas Armadas hacia las agitaciones sociales o las actividades subversivas depende de las directivas del poder político, pero también de los contenidos ideológicos de la instrucción recibida. En las democracias representativas este factor es controlado por el Parlamento; un control que en el Uruguay de los años sesenta y setenta no se ejercía. Policía y Fuerzas Armadas fueron conformándose como cuerpos separados dentro del Estado, con dinámicas propias en las que incidía el adoctrinamiento impartido por instructores extranjeros.

En las declaraciones públicas de la OPS, el Programa de Seguridad Pública se proponía capacitar a la Policía para que defendiera más eficazmente la ley, humanizando sus procedimientos. La naturaleza de esta humanización puede verificarse en el número de torturados, heridos, muertos y desaparecidos que fueron dejando a su paso oficiales uruguayos adiestrados por el PSP.

Uruguay sufrió en estos años un proceso de "brutalización de la política" al que no fueron ajenos los instructores estadounidenses. Las fuerzas policiales fueron orientadas desde 1965 para que adquirieran una creciente indiferencia por los derechos humanos y las vidas de sus compatriotas, proceso destinado a perpetuarse en la violencia aplicada por las Fuerzas Armadas, desde 1971 hasta el término de la dictadura.

70 Ibid., p. 16. Embajada de EEUU en Montevideo a Departamento de Estado, «Revised Analysis and Strategy Paper-Uruguay», 12.1.1972, secreto, p.23. NARA.RG59, box 2662. Este documento actualiza dos análisis previos, de agosto y diciembre de 1971, y complementa el análisis y plan de acción de la embajada para 1973-1974.

71 El Country Team encabezado por el embajador ad interim Frank Ortiz valoró con satisfacción la iniciación del proceso golpista en febrero de 1973. Véase: Embajada de EEUU en Montevideo a Departamento de Estado, "Country Analysis and Strategy Paper-Uruguay 1974-1975», secreto, 4.4.1973, NARA.RG59, box 2662. 
La participación de Estados Unidos en la represión interna no se limitó al suministro de asistencia técnica, armas y equipos. Los funcionarios del PSP y del MILGRP colaboraron personalmente en la actividad represiva, planificando, supervisando y orientando a los cuerpos de seguridad. La documentación del Departamento de Estado y las fuentes testimoniales revelan que los funcionarios estadounidenses no sólo encubrieron todo tipo de excesos de las fuerzas policiales, sino que directamente los promovieron. Los antecesores de Mitrione cooperaron directamente en la ofensiva contra los movimientos estudiantil y sindical e impulsaron todos los abusos cometidos en la represión de masas. A partir de entonces, las violaciones de los derechos humanos, la tortura, aunque ya existentes, se volvieron rutinarias. 